\title{
Coefficient Estimates for Initial Taylor-Maclaurin Coefficients for a Subclass of Analytic and Bi-Univalent Functions Defined by Al-Oboudi Differential Operator
}

\author{
Serap Bulut \\ Civil Aviation College, Kocaeli University, Arslanbey Campus, 41285 Izmit-Kocaeli, Turkey \\ Correspondence should be addressed to Serap Bulut; bulutserap@yahoo.com \\ Received 5 August 2013; Accepted 7 October 2013 \\ Academic Editors: H. Bulut and J. Park \\ Copyright (C) 2013 Serap Bulut. This is an open access article distributed under the Creative Commons Attribution License, which \\ permits unrestricted use, distribution, and reproduction in any medium, provided the original work is properly cited. \\ We introduce and investigate an interesting subclass $\mathscr{N}_{\Sigma}^{\lambda, \delta}(n, \beta ; h)$ of analytic and bi-univalent functions in the open unit disk $\mathbb{U}$. \\ For functions belonging to the class $\mathcal{N}_{\Sigma}^{\lambda, \delta}(n, \beta ; h)$, we obtain estimates on the first two Taylor-Maclaurin coefficients $\left|a_{2}\right|$ and $\left|a_{3}\right|$.
}

\section{Introduction}

Let $\mathbb{R}=(-\infty, \infty)$ be the set of real numbers, $\mathbb{C}$ the set of complex numbers, and

$$
\mathbb{N}:=\{1,2,3, \ldots\}=\mathbb{N}_{0} \backslash\{0\}
$$

the set of positive integers.

Let $\mathscr{A}$ denote the class of all functions of the form

$$
f(z)=z+\sum_{k=2}^{\infty} a_{k} z^{k}
$$

which are analytic in the open unit disk

$$
\mathbb{U}=\{z: z \in \mathbb{C} \text { and }|z|<1\} .
$$

We also denote by $\delta$ the class of all functions in the normalized analytic function class $\mathscr{A}$ which are univalent in $\mathbb{U}$.

For two functions $f$ and $g$, analytic in $\mathbb{U}$, we say that the function $f$ is subordinate to $g$ in $\mathbb{U}$ and write

$$
f(z) \prec g(z) \quad(z \in \mathbb{U}),
$$

if there exists a Schwarz function $\omega$, which is analytic in $\mathbb{U}$ with

$$
\omega(0)=0, \quad|\omega(z)|<1, \quad(z \in \mathbb{U})
$$

such that

$$
f(z)=g(\omega(z)), \quad(z \in \mathbb{U}) .
$$

Indeed, it is known that

$$
\begin{gathered}
f(z) \prec g(z), \quad(z \in \mathbb{U}) \Longrightarrow f(0)=g(0), \\
f(\mathbb{U}) \subset g(\mathbb{U}) .
\end{gathered}
$$

Furthermore, if the function $g$ is univalent in $\mathbb{U}$, then we have the following equivalence:

$$
\begin{gathered}
f(z) \prec g(z), \quad(z \in \mathbb{U}) \Longleftrightarrow f(0)=g(0), \\
f(\mathbb{U}) \subset g(\mathbb{U}) .
\end{gathered}
$$
ator:

For $f \in \mathscr{A}$, Al-Oboudi [1] introduced the following oper-

$$
\begin{gathered}
D_{\delta}^{0} f(z)=f(z), \\
D_{\delta}^{1} f(z)=(1-\delta) f(z)+\delta z f^{\prime}(z)=: D_{\delta} f(z), \quad(\delta \geq 0),
\end{gathered}
$$

$$
D_{\delta}^{n} f(z)=D_{\delta}\left(D_{\delta}^{n-1} f(z)\right), \quad(n \in \mathbb{N}) .
$$


If $f$ is given by (2), then from (10) and (11) we see that

$$
D_{\delta}^{n} f(z)=z+\sum_{k=2}^{\infty}[1+(k-1) \delta]^{n} a_{k} z^{k}, \quad\left(n \in \mathbb{N}_{0}\right),
$$

with $D_{\delta}^{n} f(0)=0$. When $\delta=1$, we get Sălăgean's differential operator $D_{1}^{n}=D^{n}$, [2].

Since univalent functions are one-to-one, they are invertible and the inverse functions need not be defined on the entire unit disk $\mathbb{U}$. In fact, the Koebe one-quarter theorem [3] ensures that the image of $\mathbb{U}$ under every univalent function $f \in \mathcal{S}$ contains a disk of radius $1 / 4$. Thus every function $f \in \mathscr{A}$ has an inverse $f^{-1}$, which is defined by

$$
\begin{gathered}
f^{-1}(f(z))=z \quad(z \in \mathbb{U}), \\
f\left(f^{-1}(w)\right)=w \quad\left(|w|<r_{0}(f) ; r_{0}(f) \geq \frac{1}{4}\right) .
\end{gathered}
$$

In fact, the inverse function $f^{-1}$ is given by

$$
\begin{aligned}
f^{-1}(w)= & w-a_{2} w^{2}+\left(2 a_{2}^{2}-a_{3}\right) w^{3} \\
& -\left(5 a_{2}^{3}-5 a_{2} a_{3}+a_{4}\right) w^{4}+\cdots .
\end{aligned}
$$

A function $f \in \mathscr{A}$ is said to be bi-univalent in $\mathbb{U}$ if both $f$ and $f^{-1}$ are univalent in $\mathbb{U}$. Let $\Sigma$ denote the class of biunivalent functions in $\mathbb{U}$ given by (2). For a brief history and interesting examples of functions in the class $\Sigma$, see [4] (see also $[5,6])$. In fact, the aforecited work of Srivastava et al. [4] essentially revived the investigation of various subclasses of the bi-univalent function class $\Sigma$ in recent years; it was followed by such works as those by Frasin and Aouf [7], Porwal and Darus [8], and others (see, e.g., [9-17]).

Motivated by the abovementioned works, we define the following subclass of function class $\Sigma$.

Definition 1. Let $h: \mathbb{U} \rightarrow \mathbb{C}$ be a convex univalent function such that

$$
h(0)=1, \quad h(\bar{z})=\overline{h(z)} \quad(z \in \mathbb{U} ; \mathfrak{R}(h(z))>0) .
$$

A function $f$, defined by (2), is said to be in the class $\mathscr{N}_{\Sigma}^{\lambda, \delta}(n, \beta ; h)$ if the following conditions are satisfied:

$$
\begin{aligned}
& f \in \Sigma \text {, } \\
& e^{i \beta}\left((1-\lambda) \frac{D_{\delta}^{n} f(z)}{z}+\lambda\left(D_{\delta}^{n} f(z)\right)^{\prime}\right) \prec h(z) \cos \beta+i \sin \beta \\
& (z \in \mathbb{U}) \text {, } \\
& e^{i \beta}\left((1-\lambda) \frac{D_{\delta}^{n} g(w)}{w}+\lambda\left(D_{\delta}^{n} g(w)\right)^{\prime}\right) \\
& \prec h(w) \cos \beta+i \sin \beta \quad(w \in \mathbb{U}),
\end{aligned}
$$

where $\beta \in(-\pi / 2, \pi / 2), \lambda \geq 1$, the function $g$ is given by

$$
\begin{aligned}
g(w)= & w-a_{2} w^{2}+\left(2 a_{2}^{2}-a_{3}\right) w^{3} \\
& -\left(5 a_{2}^{3}-5 a_{2} a_{3}+a_{4}\right) w^{4}+\cdots,
\end{aligned}
$$

and $D_{\delta}^{n}$ is the Al-Oboudi differential operator.
Remark 2. If we set

$$
h(z)=\frac{1+A z}{1+B z} \quad(-1 \leq B<A \leq 1)
$$

in Definition 1, then the class $\mathscr{N}_{\Sigma}^{\lambda, \delta}(n, \beta ; h)$ reduces to the class denoted by $\mathscr{N}_{\Sigma}^{\lambda, \delta}(n, \beta ; A, B)$ which is the subclass of the functions $f \in \sum$ satisfying

$$
\begin{aligned}
& e^{i \beta}(\left.(1-\lambda) \frac{D_{\delta}^{n} f(z)}{z}+\lambda\left(D_{\delta}^{n} f(z)\right)^{\prime}\right) \\
& \prec \frac{1+A z}{1+B z} \cos \beta+i \sin \beta \quad(z \in \mathbb{U}), \\
& e^{i \beta}\left((1-\lambda) \frac{D_{\delta}^{n} g(w)}{w}+\lambda\left(D_{\delta}^{n} g(w)\right)^{\prime}\right) \\
& \prec \frac{1+A w}{1+B w} \cos \beta+i \sin \beta \quad(w \in \mathbb{U}),
\end{aligned}
$$

where $\beta \in(-\pi / 2, \pi / 2), \lambda \geq 1$, the function $g$ is defined by (17), and $D_{\delta}^{n}$ is the Al-Oboudi differential operator.

Remark 3. If we set

$$
h(z)=\frac{1+(1-2 \alpha) z}{1-z} \quad(0 \leq \alpha<1)
$$

in Definition 1, then the class $\mathcal{N}_{\Sigma}^{\lambda, \delta}(n, \beta ; h)$ reduces to the class denoted by $\mathcal{N}_{\Sigma}^{\lambda, \delta}(n, \beta, \alpha)$ which is the subclass of the functions $f \in \sum$ satisfying

$$
\begin{array}{r}
\mathfrak{R}\left\{e^{i \beta}\left((1-\lambda) \frac{D_{\delta}^{n} f(z)}{z}+\lambda\left(D_{\delta}^{n} f(z)\right)^{\prime}\right)\right\}> \\
>\cos \beta \\
(z \in \mathbb{U}), \\
\mathfrak{R}\left\{e^{i \beta}\left((1-\lambda) \frac{D_{\delta}^{n} g(w)}{w}+\lambda\left(D_{\delta}^{n} g(w)\right)^{\prime}\right)\right\} \begin{array}{c}
>\cos \beta \\
(w \in \mathbb{U}),
\end{array}
\end{array}
$$

where $\beta \in(-\pi / 2, \pi / 2), \lambda \geq 1$, the function $g$ is defined by (17), and $D_{\delta}^{n}$ is the Al-Oboudi differential operator.

Remark 4. If we set

$$
\delta=1, \quad h(z)=\frac{1+(1-2 \alpha) z}{1-z} \quad(0 \leq \alpha<1)
$$

in Definition 1, then the class $\mathscr{N}_{\Sigma}^{\lambda, \delta}(n, \beta ; h)$ reduces to the class denoted by $\mathcal{N}_{\Sigma}^{\lambda}(n, \beta, \alpha)$ which is the subclass of the functions $f \in \Sigma$ satisfying

$$
\begin{aligned}
& \mathfrak{R}\left\{e^{i \beta}\left((1-\lambda) \frac{D^{n} f(z)}{z}+\lambda\left(D^{n} f(z)\right)^{\prime}\right)\right\}>\alpha \cos \beta \\
& (z \in \mathbb{U}), \\
& \mathfrak{R}\left\{e^{i \beta}\left((1-\lambda) \frac{D^{n} g(w)}{w}+\lambda\left(D^{n} g(w)\right)^{\prime}\right)\right\}>\alpha \cos \beta
\end{aligned}
$$$$
(w \in \mathbb{U}),
$$

where $\beta \in(-\pi / 2, \pi / 2), \lambda \geq 1$, the function $g$ is defined by (17), and $D^{n}$ is the Sălǎgean differential operator. 
Remark 5. If we set

$$
n=0, \quad h(z)=\frac{1+(1-2 \alpha) z}{1-z} \quad(0 \leq \alpha<1)
$$

in Definition 1 , then the class $\mathscr{N}_{\Sigma}^{\lambda, \delta}(n, \beta ; h)$ reduces to the class denoted by $\mathcal{N} \mathscr{P}_{\Sigma}^{\lambda}(\beta, \alpha)$ which is the subclass of the functions $f \in \Sigma$ satisfying

$$
\begin{aligned}
& \mathfrak{R}\left\{e^{i \beta}\left((1-\lambda) \frac{f(z)}{z}+\lambda f^{\prime}(z)\right)\right\}>\alpha \cos \beta \quad(z \in \mathbb{U}), \\
& \mathfrak{R}\left\{e^{i \beta}\left((1-\lambda) \frac{g(w)}{w}+\lambda g^{\prime}(w)\right)\right\}>\alpha \cos \beta \quad(w \in \mathbb{U}),
\end{aligned}
$$

where $\beta \in(-\pi / 2, \pi / 2), \lambda \geq 1$, and the function $g$ is defined by (17).

\section{Remark 6. If we set}

$$
n=0, \quad \lambda=1, \quad h(z)=\frac{1+(1-2 \alpha) z}{1-z} \quad(0 \leq \alpha<1)
$$

in Definition 1, then the class $\mathcal{N}_{\Sigma}^{\lambda, \delta}(n, \beta ; h)$ reduces to the class denoted by $\mathcal{N} \mathscr{P}_{\Sigma}(\beta, \alpha)$ which is the subclass of the functions $f \in \sum$ satisfying

$$
\begin{array}{cc}
\mathfrak{R}\left\{e^{i \beta} f^{\prime}(z)\right\}>\alpha \cos \beta & (z \in \mathbb{U}), \\
\mathfrak{R}\left\{e^{i \beta} g^{\prime}(w)\right\}>\alpha \cos \beta & (w \in \mathbb{U}),
\end{array}
$$

where $\beta \in(-\pi / 2, \pi / 2)$ and the function $g$ is defined by (17).

We note that

$$
\begin{gathered}
\mathcal{N} \mathscr{P}_{\Sigma}^{\lambda}(n, 0, \alpha)=\mathscr{H}_{\Sigma}(n, \alpha, \lambda) \quad(\text { see }[8]), \\
\mathcal{N} \mathscr{P}_{\Sigma}^{\lambda}(0, \alpha)=\mathscr{B}_{\Sigma}(\alpha, \lambda) \quad(\text { see }[7]), \\
\mathcal{N} \mathscr{P}_{\Sigma}(0, \alpha)=\mathscr{H}_{\Sigma}(\alpha) \quad(\text { see }[4]) .
\end{gathered}
$$

Firstly, in order to derive our main results, we need the following lemma.

Lemma 7 (see [18]). Let the function $h(z)$ given by

$$
h(z)=\sum_{n=1}^{\infty} B_{n} z^{n}
$$

be convex in $\mathbb{U}$. Suppose also that the function $\varphi(z)$ given by

$$
\varphi(z)=\sum_{n=1}^{\infty} c_{n} z^{n}
$$

is holomorphic in $\mathbb{U}$. If $\varphi(z) \prec h(z)(z \in \mathbb{U})$, then

$$
\left|c_{n}\right| \leq\left|B_{1}\right| \quad(n \in \mathbb{N}) \text {. }
$$

The object of the present paper is to find estimates on the Taylor-Maclaurin coefficients $\left|a_{2}\right|$ and $\left|a_{3}\right|$ for functions in this new subclass $\mathscr{N} \mathscr{P}_{\Sigma}^{\lambda, \delta}(n, \beta ; h)$ of the function class $\Sigma$.

\section{A Set of General Coefficient Estimates}

In this section, we state and prove our general results involving the bi-univalent function class $\mathscr{N}_{\Sigma}^{\lambda, \delta}(n, \beta ; h)$ given by Definition 1.

Theorem 8. Let the function $f(z)$ given by the Taylor-Maclaurin series expansion (2) be in the function class

$$
\mathcal{N}_{\Sigma}^{\lambda, \delta}(n, \beta ; h) \quad\left(\beta \in(-\pi / 2, \pi / 2), \lambda \geq 1, \delta \geq 0, n \in \mathbb{N}_{0}\right)
$$

with

$$
h(z)=1+B_{1} z+B_{2} z^{2}+\cdots .
$$

Then

$$
\left|a_{2}\right| \leq \min \left\{\frac{\left|B_{1}\right| \cos \beta}{(1+\delta)^{n}(1+\lambda)}, \sqrt{\frac{\left|B_{1}\right| \cos \beta}{(1+2 \delta)^{n}(1+2 \lambda)}}\right\},
$$

$$
\left|a_{3}\right| \leq \frac{\left|B_{1}\right| \cos \beta}{(1+2 \delta)^{n}(1+2 \lambda)} .
$$

Proof. It follows from (16) that

$$
\begin{array}{r}
e^{i \beta}\left((1-\lambda) \frac{D_{\delta}^{n} f(z)}{z}+\lambda\left(D_{\delta}^{n} f(z)\right)^{\prime}\right) \\
=p(z) \cos \beta+i \sin \beta \quad(z \in \mathbb{U}), \\
e^{i \beta}\left((1-\lambda) \frac{D_{\delta}^{n} g(w)}{w}+\lambda\left(D_{\delta}^{n} g(w)\right)^{\prime}\right) \\
=q(w) \cos \beta+i \sin \beta \quad(w \in \mathbb{U}),
\end{array}
$$

where $p(z) \prec h(z)$ and $q(w) \prec h(w)$ have the following Taylor-Maclaurin series expansions:

$$
\begin{aligned}
& p(z)=1+p_{1} z+p_{2} z^{2}+\cdots, \\
& q(w)=1+q_{1} w+q_{2} w^{2}+\cdots,
\end{aligned}
$$

respectively. Now, upon equating the coefficients in (36) and (37), we get

$$
\begin{gathered}
e^{i \beta}(1+\delta)^{n}(1+\lambda) a_{2}=p_{1} \cos \beta, \\
e^{i \beta}(1+2 \delta)^{n}(1+2 \lambda) a_{3}=p_{2} \cos \beta, \\
-e^{i \beta}(1+\delta)^{n}(1+\lambda) a_{2}=q_{1} \cos \beta, \\
e^{i \beta}\left[-(1+2 \delta)^{n}(1+2 \lambda) a_{3}+2(1+2 \delta)^{n}(1+2 \lambda) a_{2}^{2}\right] \\
=q_{2} \cos \beta .
\end{gathered}
$$

From (40) and (42), we obtain

$$
\begin{gathered}
p_{1}=-q_{1}, \\
2 e^{2 i \beta}(1+\delta)^{2 n}(1+\lambda)^{2} a_{2}^{2}=\left(p_{1}^{2}+q_{1}^{2}\right) \cos ^{2} \beta .
\end{gathered}
$$


Also, from (41) and (43), we find that

$$
a_{2}^{2}=\frac{e^{-i \beta}\left(p_{2}+q_{2}\right) \cos \beta}{2(1+2 \delta)^{n}(1+2 \lambda)}
$$

Since $p, q \in h(\mathbb{U})$, according to Lemma 7, we immediately have

$$
\begin{aligned}
& \left|p_{k}\right|=\left|\frac{p^{(k)}(0)}{k !}\right| \leq\left|B_{1}\right| \quad(k \in \mathbb{N}), \\
& \left|q_{k}\right|=\left|\frac{q^{(k)}(0)}{k !}\right| \leq\left|B_{1}\right| \quad(k \in \mathbb{N}) .
\end{aligned}
$$

Applying (47) and Lemma 7 for the coefficients $p_{1}, p_{2}, q_{1}$, and $q_{2}$, from the equalities (45) and (46), we obtain

$$
\begin{aligned}
\left|a_{2}\right|^{2} & \leq \frac{\left|B_{1}\right|^{2} \cos ^{2} \beta}{(1+\delta)^{2 n}(1+\lambda)^{2}}, \\
\left|a_{2}\right|^{2} & \leq \frac{\left|B_{1}\right| \cos \beta}{(1+2 \delta)^{n}(1+2 \lambda)},
\end{aligned}
$$

respectively. So we get the desired estimate on the coefficient $\left|a_{2}\right|$ as asserted in (34).

Next, in order to find the bound on the coefficient $\left|a_{3}\right|$, we subtract (43) from (41). We thus get

$$
\begin{gathered}
2(1+2 \delta)^{n}(1+2 \lambda) a_{3}-2(1+2 \delta)^{n}(1+2 \lambda) a_{2}^{2} \\
=e^{-i \beta}\left(p_{2}-q_{2}\right) \cos \beta .
\end{gathered}
$$

Upon substituting the value of $a_{2}^{2}$ from (45) into (50), it follows that

$$
a_{3}=\frac{e^{-2 i \beta}\left(p_{1}^{2}+q_{1}^{2}\right) \cos ^{2} \beta}{2(1+\delta)^{2 n}(1+\lambda)^{2}}+\frac{e^{-i \beta}\left(p_{2}-q_{2}\right) \cos \beta}{2(1+2 \delta)^{n}(1+2 \lambda)}
$$

So we get

$$
\left|a_{3}\right| \leq \frac{\left|B_{1}\right|^{2} \cos ^{2} \beta}{(1+\delta)^{2 n}(1+\lambda)^{2}}+\frac{\left|B_{1}\right| \cos \beta}{(1+2 \delta)^{n}(1+2 \lambda)} .
$$

On the other hand, upon substituting the value of $a_{2}^{2}$ from (46) into (50), it follows that

$$
a_{3}=\frac{e^{-i \beta}\left(p_{2}+q_{2}\right) \cos \beta}{2(1+2 \delta)^{n}(1+2 \lambda)}+\frac{e^{-i \beta}\left(p_{2}-q_{2}\right) \cos \beta}{2(1+2 \delta)^{n}(1+2 \lambda)} .
$$

And we get

$$
\left|a_{3}\right| \leq \frac{\left|B_{1}\right| \cos \beta}{(1+2 \delta)^{n}(1+2 \lambda)}
$$

Comparing the inequalities in (52) and (54) completes the proof of Theorem 8 .

\section{Corollaries and Consequences}

By setting

$$
h(z)=\frac{1+A z}{1+B z} \quad(-1 \leq B<A \leq 1)
$$

in Theorem 8, we have the following corollary.

Corollary 9. Let the function $f(z)$ given by the TaylorMaclaurin series expansion (2) be in the function class

$$
\begin{array}{r}
\mathcal{N}_{\Sigma}^{\lambda, \delta}(n, \beta ; A, B) \\
\left(\beta \in(-\pi / 2, \pi / 2), \lambda \geq 1, \delta \geq 0,-1 \leq B<A \leq 1, n \in \mathbb{N}_{0}\right) .
\end{array}
$$

Then

$$
\begin{gathered}
\left|a_{2}\right| \leq \min \left\{\frac{(A-B) \cos \beta}{(1+\delta)^{n}(1+\lambda)}, \sqrt{\frac{(A-B) \cos \beta}{(1+2 \delta)^{n}(1+2 \lambda)}}\right\}, \\
\left|a_{3}\right| \leq \frac{(A-B) \cos \beta}{(1+2 \delta)^{n}(1+2 \lambda)} .
\end{gathered}
$$

By setting

$$
h(z)=\frac{1+(1-2 \alpha) z}{1-z} \quad(0 \leq \alpha<1)
$$

in Theorem 8, we have the following corollary.

Corollary 10. Let the function $f(z)$ given by the TaylorMaclaurin series expansion (2) be in the function class

$$
\begin{array}{r}
\mathcal{N}_{\Sigma}^{\lambda, \delta}(n, \beta, \alpha) \\
\left(\beta \in(-\pi / 2, \pi / 2), \lambda \geq 1, \delta \geq 0,0 \leq \alpha<1, n \in \mathbb{N}_{0}\right) .
\end{array}
$$

Then

$$
\begin{gathered}
\left|a_{2}\right| \leq \min \left\{\frac{2(1-\alpha) \cos \beta}{(1+\delta)^{n}(1+\lambda)}, \sqrt{\frac{2(1-\alpha) \cos \beta}{(1+2 \delta)^{n}(1+2 \lambda)}}\right\}, \\
\left|a_{3}\right| \leq \frac{2(1-\alpha) \cos \beta}{(1+2 \delta)^{n}(1+2 \lambda)} .
\end{gathered}
$$

By setting

$$
\delta=1, \quad h(z)=\frac{1+(1-2 \alpha) z}{1-z} \quad(0 \leq \alpha<1)
$$

in Theorem 8, we have the following corollary.

Corollary 11. Let the function $f(z)$ given by the TaylorMaclaurin series expansion (2) be in the function class

$$
\begin{array}{r}
\mathcal{N}_{\Sigma}^{\lambda}(n, \beta, \alpha) \\
\left(\beta \in(-\pi / 2, \pi / 2), \lambda \geq 1,0 \leq \alpha<1, n \in \mathbb{N}_{0}\right) .
\end{array}
$$


Then

$$
\begin{gathered}
\left|a_{2}\right| \leq \min \left\{\frac{2(1-\alpha) \cos \beta}{2^{n}(1+\lambda)}, \sqrt{\frac{2(1-\alpha) \cos \beta}{3^{n}(1+2 \lambda)}}\right\}, \\
\left|a_{3}\right| \leq \frac{2(1-\alpha) \cos \beta}{3^{n}(1+2 \lambda)} .
\end{gathered}
$$

Remark 12. When $\beta=0$, Corollary 11 is an improvement of the following estimates obtained by Porwal and Darus [8].

Corollary 13 (see [8]). Let the function $f(z)$ given by the Taylor-Maclaurin series expansion (2) be in the function class

$$
\mathscr{H}_{\Sigma}(n, \alpha, \lambda) \quad\left(\lambda \geq 1,0 \leq \alpha<1, n \in \mathbb{N}_{0}\right) .
$$

Then

$$
\begin{gathered}
\left|a_{2}\right| \leq \sqrt{\frac{2(1-\alpha)}{3^{n}(1+2 \lambda)}}, \\
\left|a_{3}\right| \leq \frac{4(1-\alpha)^{2}}{2^{2 n}(1+\lambda)^{2}}+\frac{2(1-\alpha)}{3^{n}(1+2 \lambda)} .
\end{gathered}
$$

By setting

$$
n=0, \quad h(z)=\frac{1+(1-2 \alpha) z}{1-z} \quad(0 \leq \alpha<1)
$$

in Theorem 8 , we have the following corollary.

Corollary 14. Let the function $f(z)$ given by the TaylorMaclaurin series expansion (2) be in the function class

$$
\mathcal{N} \mathscr{P}_{\Sigma}^{\lambda}(\beta, \alpha) \quad(\beta \in(-\pi / 2, \pi / 2), \lambda \geq 1,0 \leq \alpha<1) .
$$

Then

$$
\begin{gathered}
\left|a_{2}\right| \leq \min \left\{\frac{2(1-\alpha) \cos \beta}{1+\lambda}, \sqrt{\frac{2(1-\alpha) \cos \beta}{1+2 \lambda}}\right\}, \\
\left|a_{3}\right| \leq \frac{2(1-\alpha) \cos \beta}{1+2 \lambda}
\end{gathered}
$$

Remark 15. When $\beta=0$, Corollary 14 is an improvement of the following estimates obtained by Frasin and Aouf [7].

Corollary 16 (see [7]). Let the function $f(z)$ given by the Taylor-Maclaurin series expansion (2) be in the function class

$$
\mathscr{B}_{\Sigma}(\alpha, \lambda) \quad(\lambda \geq 1,0 \leq \alpha<1) .
$$

Then

$$
\begin{gathered}
\left|a_{2}\right| \leq \sqrt{\frac{2(1-\alpha)}{1+2 \lambda}} \\
\left|a_{3}\right| \leq \frac{4(1-\alpha)^{2}}{(1+\lambda)^{2}}+\frac{2(1-\alpha)}{1+2 \lambda} .
\end{gathered}
$$

By setting

$$
n=0, \quad \lambda=1, \quad h(z)=\frac{1+(1-2 \alpha) z}{1-z} \quad(0 \leq \alpha<1)
$$

in Theorem 8, we have the following corollary.

Corollary 17. Let the function $f(z)$ given by the TaylorMaclaurin series expansion (2) be in the function class

$$
\mathscr{N} \mathscr{P}_{\Sigma}(\beta, \alpha) \quad(\beta \in(-\pi / 2, \pi / 2), 0 \leq \alpha<1) .
$$

Then

$$
\begin{gathered}
\left|a_{2}\right| \leq \min \left\{(1-\alpha) \cos \beta, \sqrt{\frac{2(1-\alpha) \cos \beta}{3}}\right\}, \\
\left|a_{3}\right| \leq \frac{2(1-\alpha) \cos \beta}{3} .
\end{gathered}
$$

Remark 18. When $\beta=0$, Corollary 17 is an improvement of the following estimates obtained by Srivastava et al. [4].

Corollary 19 (see [4]). Let the function $f(z)$ given by the Taylor-Maclaurin series expansion (2) be in the function class

$$
\mathscr{H}_{\Sigma}(\alpha) \quad(0 \leq \alpha<1) .
$$

Then

$$
\begin{gathered}
\left|a_{2}\right| \leq \sqrt{\frac{2(1-\alpha)}{3},} \\
\left|a_{3}\right| \leq \frac{(1-\alpha)(5-3 \alpha)}{3} .
\end{gathered}
$$

\section{References}

[1] F. M. Al-Oboudi, "On univalent functions defined by a generalized Sălăgean operator," International Journal of Mathematics and Mathematical Sciences, vol. 2004, no. 27, pp. 1429-1436, 2004.

[2] G. S. Sǎlăgean, "Subclasses of univalent functions," in Complex Analysis-Fifth Romanian-Finnish Seminar, Part 1 (Bucharest, 1981), vol. 1013 of Lecture Notes in Mathematics, pp. 362-372, Springer, Berlin, Germany, 1983.

[3] P. L. Duren, Univalent Functions, vol. 259 of Grundlehren der Mathematischen Wissenschaften, Springer, New York, NY, USA, 1983.

[4] H. M. Srivastava, A. K. Mishra, and P. Gochhayat, "Certain subclasses of analytic and bi-univalent functions," Applied Mathematics Letters, vol. 23, no. 10, pp. 1188-1192, 2010.

[5] D. A. Brannan and T. S. Taha, "On some classes of bi-univalent functions," in Mathematical Analysis and Its Applications, S. M. Mazhar, A. Hamoui, and N. S. Faour, Eds., vol. 3 of KFAS Proceedings Series, pp. 53-60, Pergamon Press, Elsevier Science, Oxford, UK, 1988.

[6] D. A. Brannan and T. S. Taha, "On some classes of bi-univalent functions," Studia Universitatis Babeş-Bolyai Mathematica, vol. 31, no. 2, pp. 70-77, 1986. 
[7] B. A. Frasin and M. K. Aouf, "New subclasses of bi-univalent functions," Applied Mathematics Letters, vol. 24, no. 9, pp. 15691573, 2011.

[8] S. Porwal and M. Darus, "On a new subclass of bi-univalent functions," Journal of the Egyptian Mathematical Society, vol. 21, no. 3, pp. 190-193, 2013.

[9] S. Bulut, "Coefficient estimates for a class of analytic and biunivalent functions," Novi Sad Journal of Mathematics, vol. 43, no. 2, pp. 59-65, 2013.

[10] S. Bulut, "Coefficient estimates for new subclasses of analytic and bi-univalent functions defined by Al-Oboudi differential operator," Journal of Function Spaces and Applications, vol. 2013, Article ID 181932, 7 pages, 2013.

[11] M. Çağlar, H. Orhan, and N. Yağmur, "Coefficient bounds for new subclasses of bi-univalent functions," Filomat, vol. 27, no. 7, pp. 1165-1171, 2013.

[12] S. P. Goyal and P. Goswami, "Estimate for initial Maclaurin coefficients of bi-univalent functions for a class defined by fractional derivatives," Journal of the Egyptian Mathematical Society, vol. 20, no. 3, pp. 179-182, 2012.

[13] T. Hayami and S. Owa, "Coefficient bounds for bi-univalent functions," Pan-American Mathematical Journal, vol. 22, no. 4, pp. 15-26, 2012.

[14] H. Orhan, N. Magesh, and V. K. Balaji, "Initial coefficient bounds for a general class of bi-univalent functions," Preprint.

[15] H. M. Srivastava, S. Bulut, M. Çağlar, and N. Yağmur, "Coefficient estimates for a general subclass of analytic and biunivalent functions," Filomat, vol. 27, no. 5, pp. 831-842, 2013.

[16] Q. H. Xu, Y. C. Gui, and H. M. Srivastava, "Coefficient estimates for a certain subclass of analytic and bi-univalent functions," Applied Mathematics Letters, vol. 25, no. 6, pp. 990-994, 2012.

[17] Q. H. Xu, H. G. Xiao, and H. M. Srivastava, "A certain general subclass of analytic and bi-univalent functions and associated coefficient estimate problems," Applied Mathematics and Computation, vol. 218, no. 23, pp. 11461-11465, 2012.

[18] W. Rogosinski, "On the coefficients of subordinate functions," Proceedings of the London Mathematical Society, vol. 48, no. 2, pp. 48-82, 1943. 


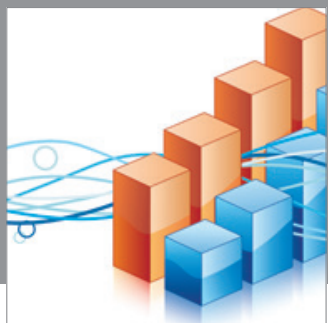

Advances in

Operations Research

mansans

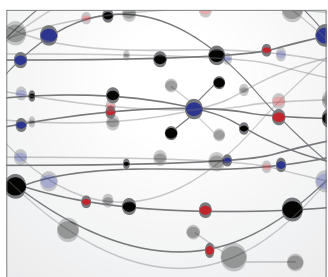

The Scientific World Journal
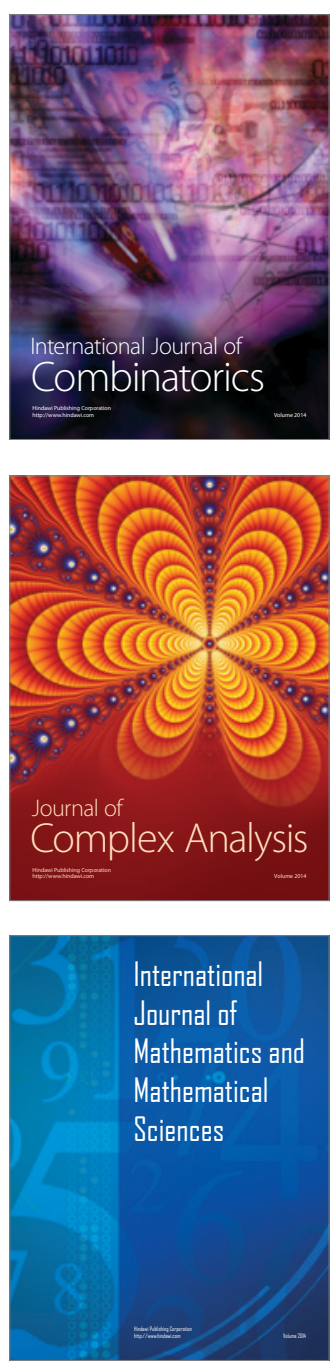
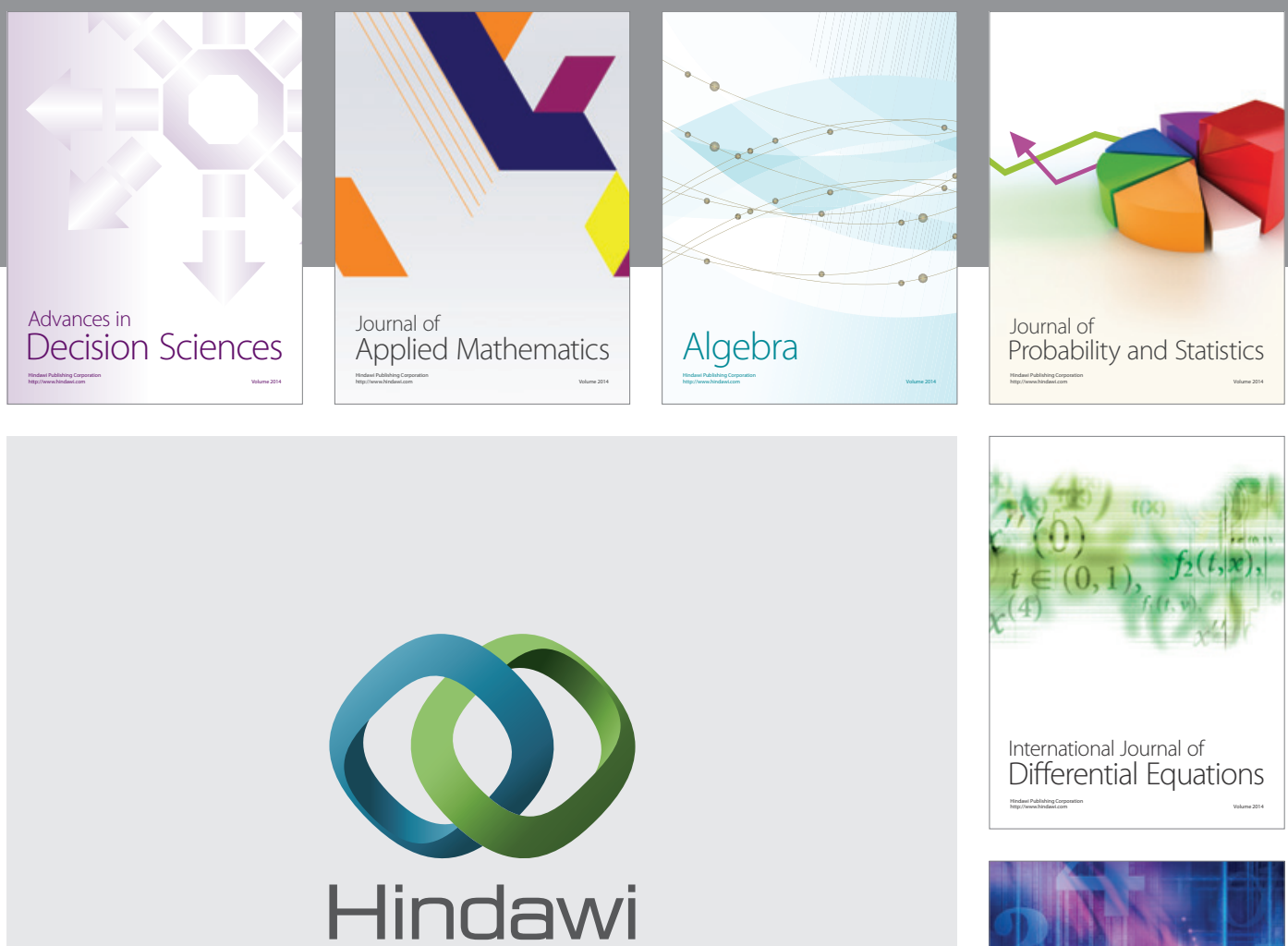

Submit your manuscripts at http://www.hindawi.com
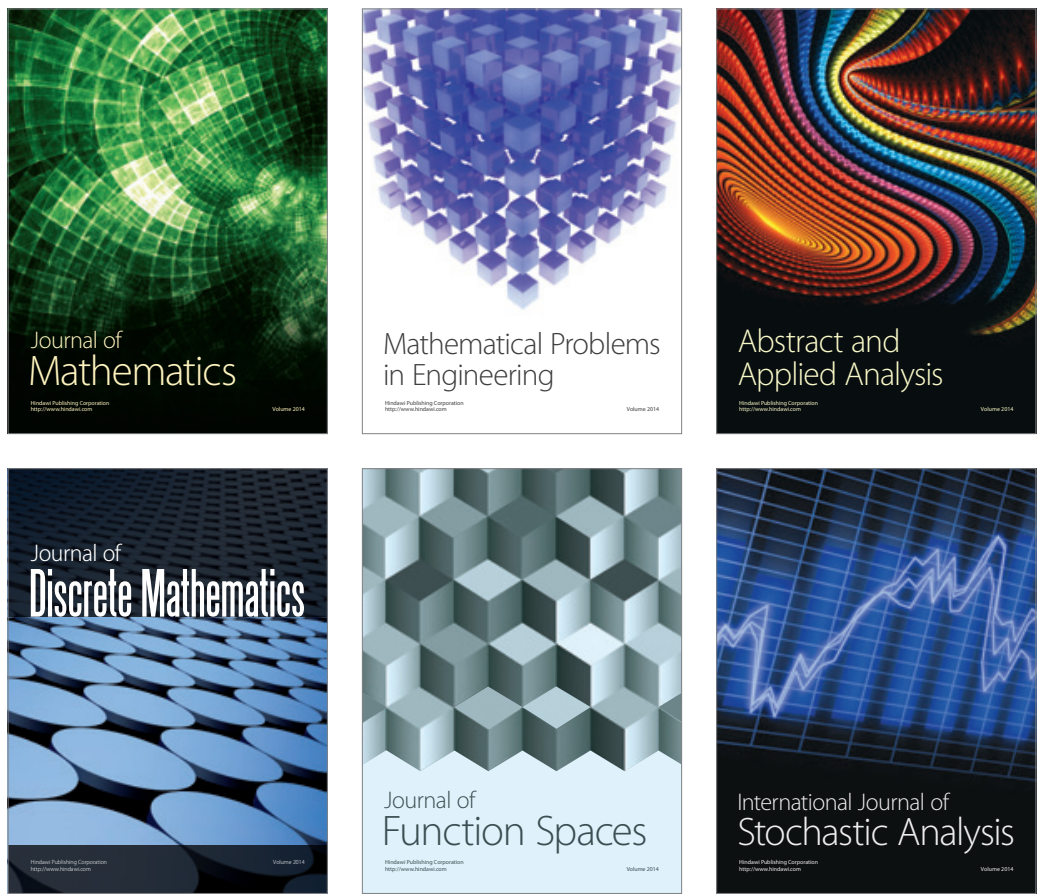

Journal of

Function Spaces

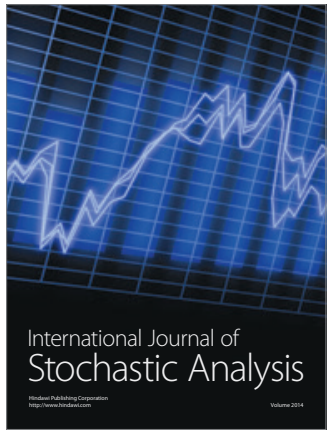

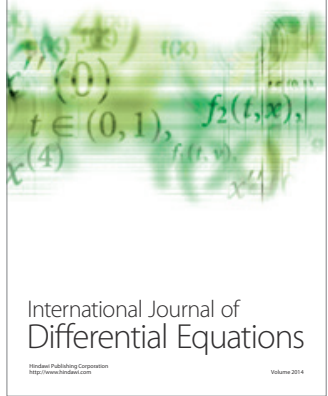
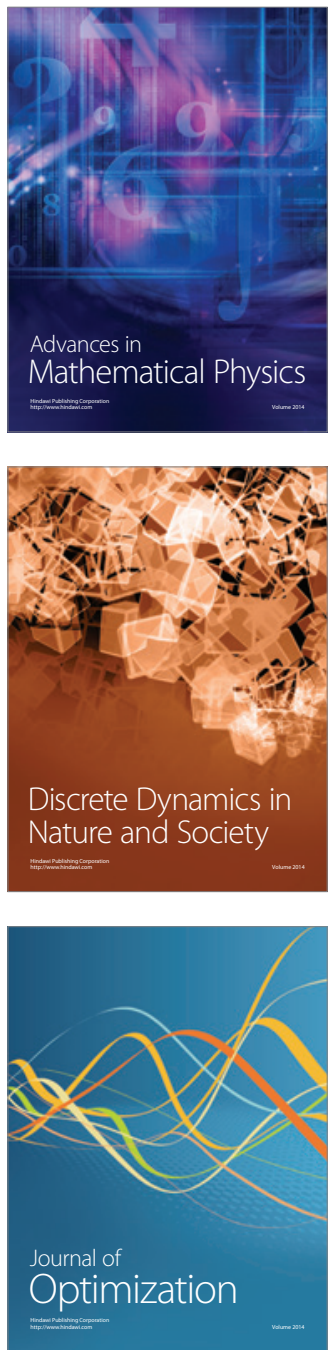